\title{
A SURVEY OF RECENT CONVERGENCE RESULTS FOR CONTINUED FRACTIONS
}

\author{
W. J. THRON*
}

1. Preliminaries. Let $a_{n}, b_{n}$ and $z$ be complex numbers and assume that $a_{n} \neq 0$ for all $n=1,2, \cdots$. We now define $s_{n}(z)=$ $a_{n} /\left(b_{n}+z\right)$, and inductively $S_{1}(z)=s_{1}(z), S_{n}(z)=S_{n-1}\left(s_{n}(z)\right)$. Then $S_{n}$ is a non-singular linear fractional transformation (l.f.t.) and hence can be written as

$$
S_{n}(z)=\frac{A_{n-1} z+A_{n}}{B_{n-1} z+B_{n}}
$$

where the $A_{n}, B_{n}$ satisfy the well known second order linear difference equations

$$
\begin{aligned}
& A_{n}=b_{n} A_{n-1}+a_{n} A_{n-2}, \\
& B_{n}=b_{n} B_{n-1}+a_{n} B_{n-2},
\end{aligned}
$$

with the initial conditions $A_{0}=0, A_{1}=a_{1}, B_{0}=1, B_{1}=b_{1}$.

The continued fraction algorithm is a function $K$ which assigns to the ordered pair of sequences $\left\langle\left\{a_{n}\right\},\left\{b_{n}\right\}\right\rangle$ the sequence $\left\{S_{n}(0)\right\}$. A continued fraction is an ordered pair $\left\langle\left\langle\left\{a_{n}\right\},\left\{b_{n}\right\}\right\rangle,\left\{S_{n}(0)\right\}\right\rangle \in K$. The quantity $S_{n}(0)=A_{n} / B_{n}$ is called the $n$th approximant of the continued fraction. One also uses the notation

$$
K\left(a_{n} / b_{n}\right)=\left\{S_{n}(0)\right\}=\left\{A_{n} / B_{n}\right\} .
$$

Note that

$$
s_{n}(\infty)=0
$$

always holds and that, if $b_{n}=1$,

$$
s_{n}(-1)=\infty
$$

is also valid. These relations play an important role in the convergence theory of continued fractions as we shall try to bring out in a later section.

In this article we are mainly concerned with convergence criteria of continued fractions whose elements $a_{n}, b_{n}$ are constants. Applications to convergence of continued fraction expansions of holomorphic

Received by the Editors February 8, 1973.

*Work supported in part by Air Force Office of Scientific Research under Grant No. AFOSR-70-1888. 
functions are possible and have been made. We illustrate the situation by stating the general parabola theorem and applying it to the convergence of certain regular $C$-fractions (for a discussion of its history see $[18]$ ).

THEOREM A. The continued fraction $K\left(a_{n} / 1\right)$ converges provided its elements $a_{n}$ lie in the parabolic region defined by

$$
\left|a_{n}\right|-\Re\left(a_{n} e^{-2 i \alpha}\right) \leqq \frac{1}{2} \cos ^{2} \alpha,-\pi / 2<\alpha<\pi / 2
$$

and at least one of the series

$$
\sum_{v=1}^{\infty}\left|\frac{a_{2} \cdots a_{2 v}}{a_{3} \cdots a_{2 v+1}}\right|, \sum_{v=2}^{\infty}\left|\frac{a_{3} \cdots a_{2 v-1}}{a_{4} \cdots a_{2 v}}\right|
$$

diverges. ((1.4) is a necessary condition for the convergence of continued fractions $\left.K\left(a_{n} / 1\right)\right)$.

THEOREM B. The regular C-fraction $1+K\left(a_{n} z / 1\right)$ converges for all $z$ inside the cardioid defined by $|z|<k(1+\cos (\arg z)), k>0$, provided for all $n \geqq 1\left|a_{n}\right|-\Re\left(a_{n}\right) \leqq 1 / 2 k$, and at least one of the series (1.4) diverges.

Let $\left\{c_{n}\right\}$ be a sequence of complex numbers satisfying $c_{n} \neq c_{n-1}$ for all $n$, but otherwise arbitrary. Then a continued fraction satisfying $S_{n}(0)=c_{n}$ for all $n$ can be found. We have $S_{1}(0)=s_{1}(0)=a_{1} / b_{1}=c_{1}$. From this equation $s_{1}$ can easily be determined. Now assume that $s_{1}$ to $s_{k-1}$ have been selected so that $S_{n}(0)=c_{n}$ is valid for $n=1, \cdots$, $k-1$. Then to have $S_{k}(0)=c_{k}$ it is necessary that $s_{k}(0)=S_{k-1}\left(c_{k}\right)$. Now $S_{k-1}^{-1}\left(c_{k}\right) \neq 0$ since $S_{k-1}(0)=c_{k-1} \neq c_{k}$, and hence $s_{k}$ can be determined. Note that $S_{k-1}^{-1}\left(c_{k}\right)=\infty$ causes no difficulty. In this case one may choose $a_{k}=1, b_{k}=0$, for example.

It follows from the above that continued fractions with complex elements having any desired speed of convergence can be constructed. Even though this fact has been known for a long time, there was a vague feeling that continued fraction expansions arising in a more natural setting would in general converge quite fast. This feeling was probably due to the well known excellent convergence behavior of regular continued fraction expansions of real numbers.

It is only in the last fifteen years, and it is this period that we shall here be mainly concerned with, that this feeling has been replaced by some facts concerning speed of convergence of certain classes of continued fractions. However, much more needs to be done.

Earlier results can be found in the books of Perron [14] and Wall [24] as well as in the survey articles $[20,22]$ of the author. 
The reader's attention is also called to the article by W. B. Jones in these proceedings. He discusses some recent results on truncation error bounds.

2. The condition $s_{n}\left(V_{n}\right) \subset V_{n-1}$. For sets $V_{n}$ in the complex plane, $n=0,1,2, \cdots$, the condition

$$
s_{n}\left(V_{n}\right) \subset V_{n-1}
$$

occurs in connection with many convergence results. This applies both to more recent results as well as to classical results. Particularly in the latter group the existence of regions satisfying (2.1) was in general not realized and in no case used explicitly in the convergence proof. We give some examples:

$$
\begin{array}{ll}
K\left(a_{n} / 1\right),\left|a_{n}\right| \leqq 1 / 4, & V_{n}=[z:|z| \leqq 1 / 2], \text { (Worpitzky) } \\
K\left(1 / b_{n}\right), \Re\left(b_{n}\right) \geqq 0, & V_{n}=[z: \Re(z) \geqq 0], \text { (Van Vleck) } \\
K\left(a_{n} / b_{n}\right),\left|b_{n}\right| \geqq\left|a_{n}\right|+1, & V_{n}=U=[z:|z| \leqq 1], \text { (Pringsheim) } \\
K\left(c_{n}{ }^{2} / 1\right),\left|\Im\left(c_{n}\right)\right| \leqq 1 / 2, & V_{n}=[z: \Re(z) \geqq-1 / 2], \text { (Scott and Wall). }
\end{array}
$$

Note that the conditions insuring the mapping requirement are in some instances less restrictive than those insuring convergence. The condition (2.1) also is present in Wall's theory of positive definite continued fractions.

In view of all this it seems natural to make the condition (2.1) the cornerstone of our investigations into the convergence behavior of continued fractions. While in the classical results the restrictions on the elements came first and the value regions were at best a byproduct, for a general approach it is desirable to start with a sequence $\left\{V_{n}\right\}$ of regions and to determine restrictions on the elements $a_{n}, b_{n}$ so that (2.1) holds for the preassigned regions $V_{n}$. More precisely, one can determine regions $E_{n}$ in the product of the complex plane with itself which are such that $\left\langle a_{n}, b_{n}\right\rangle \in E_{n}$ implies $s_{n}\left(V_{n}\right) \subset V_{n-1}$. The regions $E_{n}$ may be empty or very small. It thus becomes important to choose the $V_{n}$ so as to obtain regions $E_{n}$ as large as possible.

At this point it is convenient to introduce

$$
\mathfrak{A}=[V \text { : the boundary of } V \text { is a circle or straight line }] \text {. }
$$

The elements of $\mathfrak{K}$ are thus circular disks, half planes, or complements of circular disks. An element of $\mathfrak{A}$ may contain none, part, or all of its boundary. 
An immediate consequence of $(2.1)$ is

$$
S_{n}\left(V_{n}\right)=S_{n-1}\left(s_{n}\left(V_{n}\right)\right) \subset S_{n-1}\left(V_{n-1}\right) .
$$

Repeated application of $(2.2)$ yields

$$
S_{n}\left(V_{n}\right) \subset S_{1}\left(V_{1}\right)=s_{1}\left(V_{1}\right) \subset V_{0} .
$$

It follows that if $0 \in V_{n}$ then $S_{n}(0) \in V_{0}$. Thus if $0 \in V_{n}$ for all $n=1,2,3, \cdots$ then $V_{0}$ contains all of the approximants of the continued fraction. This information can be used to extend known convergence criteria by employing the Stieltjes-Vitali theorem (see [20] for a more detailed description of this method). This approach has led to some very general convergence results. For some of these, other proofs have since been found, but for a number of others, in particular those for which the $V_{n}$ are not elements of $\mathfrak{A}$, the Stieltjes-Vitali theorem still provides the only known proof. An example of such a result, proved by the author in 1949, is:

THEOREM C. The continued fraction $K\left(1 / b_{n}\right)$ converges provided

$$
\left|b_{2 n}\right| \geqq f\left(\arg b_{2 n}\right), \quad\left|b_{2 n-1}\right| \geqq 4 / f\left(\pi-\arg b_{2 n-1}\right),
$$

where

$$
f(\theta)=f_{0} \exp \int_{\pi / 2}^{\theta} \tan \alpha(x) d x, f_{0}>0,
$$

and $\alpha(\theta)$ is a continuous function of period $2 \pi$ satisfying the two conditions

$$
\begin{gathered}
|\alpha(\theta)|<\pi / 2-\epsilon_{1}, \epsilon_{1}>0, \\
|\alpha(\theta)-\alpha(\varphi)||| \theta-\varphi \mid<1-\epsilon_{2}, \theta \neq \varphi, \epsilon_{2}>0 .
\end{gathered}
$$

(The conditions insure that the regions bounded by the curves $r=f(\theta)$ and $r=4 / f(\pi-\theta)$ are both convex $)$.

Now let $K_{n}=S_{n}\left(V_{n}\right)$. Then it is a consequence of (2.2) that the $K_{n}$ form a nested sequence of sets. If we make the additional assumptions

$$
V_{n} \in \mathfrak{K}, n=0,1, \cdots, V_{0} \text { is a circular disk, }
$$

then it follows from (2.3) that all $K_{n}$ are circular disks. Let $R_{n}$ be the radius of $K_{n}$ Since the disks $K_{n}$ are nested $\lim R_{n}=R$ always exists. Further, if $0 \in V_{n_{k}}$ for a subsequence $\left\{n_{k}\right\}$ of natural numbers, then $S_{n_{k}}(0) \in K_{n_{k}} \subset K_{m}, m \leqq n_{k}$. Thus if $\lim S_{n_{k}}(0)=S$ exists then it must 
be in the closure of $K_{n}$ for all $n$. Hence $\left|S_{n_{k}}(0)-S\right| \leqq 2 R_{n_{k}}$, so that $R=0$ is sufficient for the convergence of $\left\{S_{n_{k}}(0)\right\}$.

Thus we have a second method for obtaining convergence criteria for certain subsequences or the whole sequence of approximants of a continued fraction. The procedure, which we shall describe in more detail in the next section, consists in obtaining sufficiently tight upper bounds on $R_{n}$ to decide whether $\lim R_{n}=0$.

3. The limit point case. Since $\lim R_{n}=R$ always exists for a nested sequence of circular disks $\left\{K_{n}\right\}$, only two, essentially different, situations can arise. If $R>0$ we speak of the limit circle case, if $R=0$ we have the limit point case. A continued fraction for which the limit circle case holds may, but need not, converge. If the limit point case holds the continued fraction must converge.

The quantities $R_{n}$ can be computed in various ways. Geometric properties of l.f.t. as well as integration, to compute the length of the circumference of the boundary of $K_{n}$, have been used. Generally it is convenient to study the ratios $R_{n} / R_{n-1}$. Since it is known that for all $n, R_{n} / R_{n-1} \leqq 1$, it is frequently sufficient to estimate $R_{n_{m}} / R_{n_{m}-1}$ for a suitable subsequence $\left\{n_{m}\right\}$. The ratio $R_{n+1} / R_{n}$ depends on a number of variables. Among these the quantity

$$
h_{n}=S_{n}^{-1}(\infty)=-B_{n} / B_{n-1}
$$

plays a particularly important role. It is of interest to note that the $h_{n}$ come up in many other contexts in continued fraction theory. So the condition $B_{n} \neq 0$, which is equivalent to $h_{n} \neq 0, \infty$, is critical in many convergence arguments. Further, Lane and Wall [11] and Hayden [4] have investigated these quantities extensively.

Recently, Field and Jones [1] deduced from the simple observation that $S_{n}\left(h_{n}\right)=\infty$, together with the assumption $\infty \notin V_{n}, n=0$, $1,2, \cdots$ the conclusion that $h_{n} \notin V_{n}$. Thus $h_{n}$ is in the complement of $V_{n}$. For continued fractions $K\left(1 / b_{n}\right)$ this information about $h_{n}$ proved to be sufficient to obtain usable results for $R_{n} / R_{n-1}$, even when all $V_{n}$ are different, a case which cannot be handled at all with procedures developed earlier. We shall now describe the earlier method.

If $b_{n}=1$, then $s_{n}^{-1}(z)=u \circ s_{n} \circ u^{-1}(z)$, where $u=-1-z$. If $a_{n}=1$ then $s_{n}^{-1}(z)=u \circ s_{n} \circ u^{-1}(z)$, provided $u=-1 / z$. In either case

$$
\begin{aligned}
& h_{n}=S_{n}{ }^{-1}(\infty)=s_{n}{ }^{-1} \circ s_{n-1}^{-1} \circ \cdots \circ s_{1}^{-1}(\infty) \\
& =u \circ s_{n} \circ s_{n-1} \circ \cdots \circ s_{1}\left(u^{-1}(\infty)\right) \text {. }
\end{aligned}
$$


For $K\left(a_{n} / 1\right)$ we thus have

$$
h_{n}=-1-s_{n} \circ s_{n-1} \circ \cdots \circ s_{2}(0) \text {, }
$$

and for $K\left(1 / b_{n}\right)$

$$
h_{n}=-1 / s_{n} \circ s_{n-1} \circ \cdots \circ s_{1}(0) .
$$

If one now makes the additional assumption on the sequence $\left\{V_{n}\right\}$ that

$$
V_{n}=V_{m} \text { for } n \equiv m(\bmod 2),
$$

then one obtains $h_{n} \in u\left(V_{n+1}\right)$ as a first approximation to the location of $h_{n}$. In certain cases this is enough information to conclude that $\lim R_{n}=0$. However, in the endeavors to obtain largest twinconvergence regions additional restrictions on $h_{n}$ had to be derived. These are quite delicate and depend on the fact that $u\left(h_{n}\right)$ is the image of 0 under $n$ (or $n-1$ ) transformations $s$. In the cases under consideration one was then able to conclude that $u\left(h_{n}\right)$ had to stay a certain distance, depending on $n$, away from the boundary of $V_{n}$. These ideas were developed in the articles [16], [18], [19], [13] and [12]. The result of Lange in [12] is as follows.

TheOREM D. The continued fraction $K\left(c_{n}{ }^{2} / 1\right)$ converges provided

$$
\left|c_{2 n-1} \pm i a\right| \leqq \rho,\left|c_{2 n} \pm i(1+a)\right| \geqq \rho,
$$

where $|a|<\rho<|1+a|$. Moreover

$$
V_{2 n}=[z:|z-a| \leqq \rho], V_{2 n-1}=[z:|z+(1+a)| \geqq \rho] .
$$

Further,

$$
\left|h_{2 n-1}+\left(1+a k_{n}\right)\right| \leqq \rho k_{n}
$$

where $k_{n}=(n-1) /(n-1+b(a, \rho)), b(a, \rho)>0$. Finally,

$$
\begin{aligned}
\frac{R_{2 n}}{R_{2 n-1}} & =\frac{\left|a_{2 n}\right|\left(\rho^{2}-\left|1+a h_{2 n-1}\right|^{2}\right)}{\left|(1+a) k_{2 n-1}-a_{2 n}\right|^{2}-\rho^{2}\left|h_{2 n-1}\right|^{2}} \\
& \leqq 1-\frac{d(a, \rho)}{h(a, \rho)+(n-1) k(a, \rho)},
\end{aligned}
$$

where $d(a, \rho)>0, h(a, \rho)>0, k(a, \rho)>0$.

While in the articles cited above the aim was to get the largest possible convergence regions, even at the price of very slow convergence $\left(R_{n} \sim n^{-\epsilon}\right.$ in some instances), there has been recent work by Sweezy and Thron [17], Jones and Snell [7], and Field and Jones 
[1], directed towards getting faster convergence for suitably restricted continued fractions of the form $K\left(1 / b_{n}\right)$. Results giving "geometric" speed of convergence (i.e., $R_{n}<d^{n}, 0<d<1$ ) have been obtained in all cases. Whether a higher speed of convergence can be shown to hold for substantial classes of continued fractions is at present unknown. Also, deriving better truncation error estimates for $K\left(a_{n} / 1\right)$ by restricting the $a_{n}=c_{n}{ }^{2}$ to suitable subregions of the known best regions would be a very worthwhile undertaking. Basic tools are available in the papers [12] and [19].

We conclude this section by presenting a result of Sweezy and Thron [17], which is also contained as a special case in a more general result of Field and Jones [1].

THEOREM E. The continued fraction $K\left(1 / b_{n}\right)$ converges provided

$$
\left|b_{n}+\frac{2-\epsilon}{1-\epsilon} h\right| \geqq \frac{2-\epsilon}{1-\epsilon}\left(1+h^{2}-\epsilon\right)^{1 / 2},
$$

where $h$ is an arbitrary real number and $0<\epsilon<1$. Moreover

$$
R_{n} / R_{n-1} \leqq 1-\epsilon\left(1-\left(\frac{|h|}{\left(1+h^{2}-\epsilon\right)^{1 / 2}}\right)\right) .
$$

4. Convergence of the sequence of even approximants. It has been known for a long time that for certain continued fractions the sequence of even and odd approximants (the even and odd part) may converge to different limits (for example if $b_{n}>0, \sum b_{n}<\infty$ ). Also the "fundamental inequalities" of Scott and Wall [24, p. 52] insure the convergence of the even and odd part of the continued fraction. This was the original approach to the proof of the parabola theorem for $\alpha=0$.

However, the first to derive a convergence criterion for the even part of a continued fraction only was Perron [15]. The author [19], [23] was able to improve this result as follows.

Theonem F. In $K\left(c_{n}{ }^{2} / 1\right)$ let the $c_{n}$ satisfy condition (3.1), where $\rho>|1+a|, \rho>|a|$, and

$$
\left|c_{2 n}^{2}\right| \geqq \rho^{2}-|1+a|^{2}+\epsilon, \epsilon>0,
$$

then the even part of the continued fraction converges.

We have as before $V_{2 n}=[z:|z-a| \leqq \rho]$ and $V_{2 n-1}=$ $[z:|z+a+1| \geqq \rho]$, hence the conditions $\rho>|a|$ and $\rho>|1+a|$ insure $0 \in V_{2 n}, 0 \notin V_{2 n-1}$. Thus using arguments sketched in Section 2 one is able to prove convergence of the even part but not of the 
whole continued fraction. In the case where $a=0$ an estimate for $R_{n}$ was obtained, in the general case the Stieltjes-Vitali theorem was used. Note that to get the present result some condition of the form (4.1) has to be imposed on the $c_{n}$.

Convergence results for the even part (or in some instances the odd part) of a continued fraction have also been obtained by the method which we shall discuss in the next section. For results see [10].

5. A non-constructive elementary method. In most recent work on convergence theory the continued fraction is obtained from a certain sequence of l.f.t. This leads to the question: in what ways does a sequence $\left\{S_{n}(z)\right\}$ differ from an arbitrary sequence $\left\{T_{n}(z)\right\}$ of l.f.t. The assumption

$$
T_{n}=t_{1} \circ t_{2} \circ \cdots \circ t_{n},
$$

is easily seen to be no restriction since one can set $t_{1}=T_{1}, t_{n}=$ $T_{n} \circ T_{n-1}^{-1}, n \geqq 2$. However, a restriction $t_{n}\left(U_{n}\right) \subset U_{n-1}$ proved to play a key role in obtaining useful results. A study of sequences of this kind was undertaken by the author [21] and was applied to obtaining the first elementary proof of the general parabola theorem (Theorem A). The method initiated in [21] was further refined by Hillam and Thron [6], Jones and Thron [9], [10] and Jones and Snell [8] and led not only to new proofs of old results but also to many new results. We now give a sketch of the method.

Let $S_{n}(0)$ be the approximants of a continued fraction which satisfies (2.1) with $V_{n} \in \mathfrak{R}$ for all $n \geqq 0$. By introducing l.f.t. $v_{n}$ with $v_{n}\left(V_{n}\right)=U$, where $U$ is the unit circular disk $[z:|z| \leqq 1]$, one obtains for $t_{n}=v_{n-1} \circ s_{n} \circ v_{n}^{-1}$ the normalized condition

$$
t_{n}(U) \subset U .
$$

We consider $T_{n}=t_{1} \circ t_{2} \circ \cdots \circ t_{n}$, and observe that $T_{n}=$ $v_{0} \circ S_{n} \circ v_{n}{ }^{-1}$. The conditions (1.1) and (1.2) now become

$$
t_{n}\left(\zeta_{n}\right)=\eta_{n}, t_{n}\left(\xi_{n}\right)=\omega_{n} .
$$

Knowledge of the location of $0,-1, \infty$, with respect to $V_{n}$ leads to additional conditions such as

$$
\left|\zeta_{n}\right| \geqq 1,\left|\eta_{n}\right| \leqq 1-\epsilon, \text { or }|| \xi_{n}|-1| \geqq \epsilon^{\prime}, \quad|| \omega_{n}|-1| \geqq \epsilon^{\prime},
$$

for example. The assumptions (5.2) give the $t_{n}$ a property weakly akin to their being contraction maps, a concept introduced into the theory by Hayden [4]. 
Condition (5.1) insures that the sequence $\left\{T_{n}(U)\right\}$ forms a nested sequence of circular disks. Thus we have either the limit point case and hence convergence of $\left\{T_{n}(0)\right\}$, or the limit circle case. To conditions of the form (5.2) one now adds the assumption that the limit point case does not hold (i.e., the assumption $R>0$ ) and is, in many instances able to conclude that in the limit circle case $\left\{T_{n}(0)\right\}$ converges. Since $\left\{S_{n}(0)\right\}$ is intimately related to $\left\{T_{n}(0)\right\}$, in particular if one is able to choose $v_{n}(0)=0$ (possible if $0 \in \operatorname{Int} V_{n}$ ), one frequently can draw conclusions about the convergence of $\left\{S_{n}(0)\right\}$.

The argument is completely elementary (no deep function theoretic results, such as the Stieltjes-Vitali theorem, are used) but it is not constructive and thus does not lend itself either to the determination of truncation errors or even to deciding whether the limit circle case actually occurs. It is known [21] that for continued fractions satisfying the Pringsheim criterion $\left|b_{n}\right| \geqq\left|a_{n}\right|+1$ the limit circle case can occur. In Theorem $A$ it is not as yet known whether the limit circle case is possible for $\alpha \neq 0$ (it is impossible for $\alpha=0$ ).

The advantage of the method is that it leads to a large number of convergence criteria. Many combinations of regions $V_{n}$, provided $V_{n} \in \mathfrak{R}$ and provided they are reasonably placed with respect to $0,-1, \infty$ so as to yield conditions of the form (5.2), produce convergence results. There thus arises a question about overlapping and containment of different results. Some initial steps were taken in this direction by Jones and Thron [10], much more however needs to be done.

We conclude by giving a sample of the kind of result obtained in [10]. This is a new result for which no other proof is known. Only the special case $\Gamma=0$ was previously proved in [19]. The conditions in this case become

$$
\left|a_{2 n}\right| \leqq \rho, \quad\left|a_{2 n-1}\right| \geqq 2\left(\rho-\cos \arg a_{2 n-1}\right), \quad \rho>1 .
$$

THEOREM G. The continued fraction $K\left(a_{n} / 1\right)$ converges if its elements satisfy $a_{2 n-1} \in E_{1}, a_{2 n} \in E_{2}$, where

$$
\begin{array}{r}
E_{1}=\left\{w: \rho|w|-\left|w(1+\bar{\Gamma})-(1+\Gamma)\left(\zeta^{2}-|1+\Gamma|^{2}\right)\right|\right. \\
\left.\geqq|1+\Gamma|\left(\rho^{2}-|1+\Gamma|^{2}\right)\right\}, \\
E_{2}=\left\{w:|1+\Gamma||w|+\left|w \bar{\Gamma}-\Gamma\left(|1+\Gamma|^{2}-|\Gamma|^{2}\right)\right|\right. \\
\\
\left.\quad \rho\left(|1+\Gamma|^{2}-|\Gamma|^{2}\right)\right\},
\end{array}
$$

and $|\Gamma|<|1+\Gamma|<\rho$. 


\section{REFERENCES}

1. David Field and W. B. Jones, A priori estimates for truncation errors of continued fractions $K\left(1 / b_{n}\right)$, Numer. Math. 19 (1972), 283-302.

2. T. L. Hayden, Some convergence regions for continued fractions, Math. Zeitschrift 79 (1962), 376-380.

3. —_, A convergence problem for continued fractions, Proc. Amer. Math. Soc. 14 (1963), 546-552.

4. — Continued fraction approximation to functions, Numer. Math. 7 (1965), 292-309.

5. K. L. Hillam, Some convergence criteria for continued fractions, Ph.D. Thesis, University of Colorado, 1962.

6. K. L. Hillam and W. J. Thron, A general convergence criterion for continued fractions $K\left(a_{n} / b_{n}\right)$, Proc. Amer. Math. Soc. 16 (1965), 1256-1262.

7. William B. Jones and R. I. Snell, Truncation error bounds for continued fractions, SIAM J. Numer. Anal. 6 (1969), 210-221.

8. - Sequences of convergence regions for continued fractions $K\left(a_{n} / 1\right)$, Trans. Amer. Math. Soc. 170 (1972).

9. William B. Jones and W. J. Thron, Convergence of continued fractions, Canad. J. Math. 20 (1968), 1037-1055.

10. - Twin convergence regions for continued fractions $K\left(a_{n} / 1\right)$, Trans. Amer. Math. Soc. 150 (1970), 93-119.

11. R. E. Lane and H. S. Wall, Continued fractions with absolutely convergent even and odd parts, Trans. Amer. Math. Soc. 67 (1949), 368-380.

12. L. J. Lange, On a family of twin convergence regions for continued fractions, Illinois J. Math 10 (1966), 97-108.

13. L. J. Lange and W. J. Thron, A two-parameter family of best twin convergence regions for continued fractions, Math. Zeitschrift 73 (1969), 295-311.

14. Oskar Perron, Die Lehre von den Kettenbrüchen, 3rd edition, vol. 2, Stuttgart, 1957.

15. — Über zwei Kettenbrüche von H. S. Wall, Bayer, Akad. Wiss. Math.-Nat. Kl. S.-B, (1957), 1-13.

16. Vikramaditya Singh and W. J. Thron, A family of best twin convergence regions for continued fractions, Proc. Amer. Math. Soc. 7 (1956), 277-282.

17. W. B. Sweezy and W. J. Thron, Estimates of the speed of convergence of certain continued fractions, SIAM J. Numer. Anal. 4 (1957), 254-270.

18. W. J. Thron, On parabolic convergence regions for continued fractions, Math. Zeitschrift 69 (1958), 173-182.

19. - Zwillingskonvergenzgebiete für Kettenbrüche $1+K\left(a_{n} / 1\right)$, deren eines die Kreisscheibe $\left|a_{2 n-1}\right|<\rho^{2}$ ist, Math. Zeitschrift 70 (1959), 310-344.

20. - Convergence regions for continued fractions and other infinite processes, Amer. Math. Monthly 68 (1961), 734-750.

21. - Convergence of sequences of linear fractional transformations and of continued fractions, J. Indian Math. Soc. 27 (1963), 103-127.

22. - Some results and problems in the analytic theory of continued fractions, Math. Student 32 (1964), 61-73.

23. - On the convergence of the even part of certain continued fractions, Math. Zeitschrift 85 (1964), 268-273.

24. H. S. Wall, Analytic theory of continued fractions, Princeton, 1948.

25. — Partially bounded continued fractions, Proc. Amer. Math. Soc. 7 (1956), 1090-1093.

University of Colorado, Boulder, Colorado 80302 